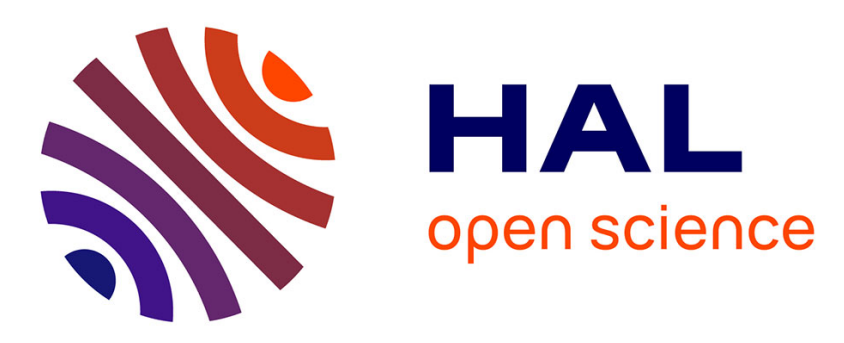

\title{
A Robustness Comparison of Measured Narrowband CSI vs RSSI for IoT Localization
}

\author{
Ahmed Abdel Ghany, Bernard Uguen, Dominique Lemur
}

\section{To cite this version:}

Ahmed Abdel Ghany, Bernard Uguen, Dominique Lemur. A Robustness Comparison of Measured Narrowband CSI vs RSSI for IoT Localization. 2020 IEEE 92nd Vehicular Technology Conference: VTC2020-Fall, Nov 2020, Victoria (virtual), Canada. 10.1109/VTC2020-Fall49728.2020.9348854 . hal-03083144

\section{HAL Id: hal-03083144 \\ https://hal.science/hal-03083144}

Submitted on 18 Dec 2020

HAL is a multi-disciplinary open access archive for the deposit and dissemination of scientific research documents, whether they are published or not. The documents may come from teaching and research institutions in France or abroad, or from public or private research centers.
L'archive ouverte pluridisciplinaire HAL, est destinée au dépôt et à la diffusion de documents scientifiques de niveau recherche, publiés ou non, émanant des établissements d'enseignement et de recherche français ou étrangers, des laboratoires publics ou privés. 


\title{
A Robustness Comparison of Measured Narrowband CSI vs RSSI for IoT Localization
}

\author{
Ahmed Abdel Ghany, Bernard Uguen, Dominique Lemur \\ Institute of Electronics and Telecommunications of Rennes (IETR) \\ University of Rennes 1 \\ Rennes, France \\ \{ahmed.abdelghany, bernard.uguen, dominique.lemur\}@univ-rennes1.fr
}

\begin{abstract}
Received Signal Strength Indicator (RSSI)-based fingerprinting is currently viewed as an important technique for the positioning capabilities in the Internet of Things (IoT). However, in the case of practical measurement, the localization methods based on RSSI are easily affected by the temporal and spatial variation, which contributes to most of the estimation errors in current systems. In this paper, the feasibility of utilizing the Channel State Information (CSI) for localization is studied, after knowing that the CSI contains information about the channel between the sender and receiver at the level of individual data subcarriers. Unlike most of the previous work, the intended approach is to use the entire subcarrier magnitudes without averaging or any reduction of the obtained narrowband CSI. Moreover, the frequency hopping in the LoRa systems should be a profit for localization by getting access to a wider band. In order to obtain a reliable basis for this approach, an outdoor measurement campaign is performed in the area of the Campus Beaulieu in Rennes to estimate the CSI of transmitted LoRa signals from different locations. For this, it is necessary for the individual channels from each different position to be appropriately different from one another to achieve significant localization gain. Hence, a comparison is done investigating the attainable evolution in the CSI at each location based on the CSI slope versus its average amplitude. In the given results, the feasibility of using the proposed technique is asserted by the drastic stability of the CSI slope over time and space, in contrary to the CSI average amplitude. This manifests the robustness of the CSI to the signal fluctuations and its more valuable rendering than the RSSI.
\end{abstract}

Index Terms-IoT, LoRa, Localization, CSI, RSSI

\section{INTRODUCTION}

Beyond the traditional voice, video and data services where data throughput is the main purpose in the context of the Internet of Things (IoT), the focus here is on low-cost deployments with large coverage areas [1]. For providing this connectivity, Low Power Wide Area Networks (LPWAN) are considered the major technology, especially the LoRaWAN whose design compromises between low energy consumption and a large communication range [2].

Localization is one of the essential features of many IoT modules due to the very nature of the data collected from the devices [3]. These usages involve location detection of assets in a warehouse, patient tracking inside the building of the hospital, and emergency personnel positioning in a disaster area. Therefore, precise localization is still a critical missing component and it has been gaining growing interest from a wide range of applications. Although equipping each sensor with a global positioning system (GPS) chip is considered a tempting option, it is not a simple solution because it requires adding a GPS tracker to a device which will increase both cost and power consumption [4]. Subsequently, a great number of researches have been done to address the domain of GPS-Free localization in IoT. These methods are investigated based on Received Signal Strength Indicator (RSSI), Angle of Arrival (AoA), Time of Arrival (ToA), Time Difference of Arrival (TDoA) and their multiple integrations [5]. For localization, these measurements are utilized by the gateways to determine their relative position relations with the source. However, using the AoA and ToA techniques always require a precise calibration or an additional hardware [6]. Therefore, these two kinds of techniques are not so practical. While TDoA is considered the most popular technique for localization as it does not require the transmitter to be synchronized with the receivers but only the gateways are required to have synchronized clocks [7]. Nevertheless, this accurate time synchronization can only be achieved by adding a GPS receiver at each gateway which needs an additional cost.

Among all the localization technologies, wireless RSSI fingerprinting has proven as an effective positioning technique due to its simplicity and deployment practicability [8]. Fingerprinting based localization avoids hardware deployment cost and effort by relying on existing network infrastructure. It just relies on the received signal strength at each gateway to localize the node. However, RSSI-based fingerprinting localization methods may have poor positioning performance as RSSI always vary due to the large signal power fluctuations both in time and space, moreover, each measured RSSI value depends on the hardware accuracy as well as the system calibration for every measurement [9]. Besides, RSSI contains coarse information so as to not fully utilize the abundant channel information in each subcarrier. Therefore, some little work has been published whose aim is to take the average value of the whole Channel State Information (CSI) subcarrier amplitudes which is proven to be more temporally stable in different environments and helps maintain the performance 
over time in comparison with RSSI [10].

In this paper, it is favorable to show the possibility to leverage CSI for improving the performance of positioning by investigating the profit of using the entire subcarrier magnitudes without averaging or any reduction. Thus, an initial measurement campaign is done to compute the narrowband CSI of transmitted LoRa signals from different locations. To achieve significant localization gain, it is necessary for the individual channels from each different position to be uncorrelated with one another. Thus, the presented short time data indicate that channel slopes with even short separated distances will be quite stable and show weak intercorrelations between them. Furthermore, the CSI in each position after a while is more correlated with itself which can achieve a significant diversity gain in comparison with the mean amplitude of the CSI. This trial allows us also to derive recommendations for the use of diversity at the receiving site in short-range outdoor-to-outdoor transmission systems, asking questions like: "At what distance must vertically polarized antennas be placed such that intercorrelation is low and hence gateway diversity is potentially beneficial for localization?" or "Can also the LoRa frequency hopping add a diversity gain for a static transmitting node scenario?"

The remainder of this document is organized as follows. Section II presents the measurement overview and Section III provides sufficient detail of the proposed post-processing algorithm. The results of the channel correlation analysis are then presented and commented in Section IV. Finally, Section V is dedicated for conclusions.

\section{System And Measurement Setup}

The main concept of the proposed experiment is transmitting repeated up-chirps signal to sense the channels consecutively at the typical uplink frequency bands, i.e 9 channels with center frequency $f_{k} \in$ $\{867.1,867.3,867.5,867.7,867.9,868.1,868.3,868.5,868.8\}$ $\mathrm{MHz}$, and $125 \mathrm{kHz}$ bandwidth. This is considered as a traditional channel sounder with a typical structure which has the Transmitter (Tx) and Receiver ( $\mathrm{Rx}$ ) placed at two different locations, as shown in Figure 1. Thus, the $\mathrm{Rx}$ antenna is fixed on the roof of the university building as shown in Figure 2a. While the Tx has a mobile structure with a laptop and a Universal Software Radio Peripheral (USRP) as it is described in Figure 2b. First, the Tx, i.e. located in specific positions within the area of the Campus Beaulieu in Rennes, should generate a signal by a laptop and transmit it using the USRP to sample the channel for a specific time interval. While the stationary Rx, whose antenna is located above the building, should receive the signal with its USRP at the same time interval of transmitting. After the Rx picks up the signal, the desktop computer stores it to perform an essential post-processing algorithm on the received signal to mitigate the imperfections and obtain the channel transfer function as detailed in the following subsection.

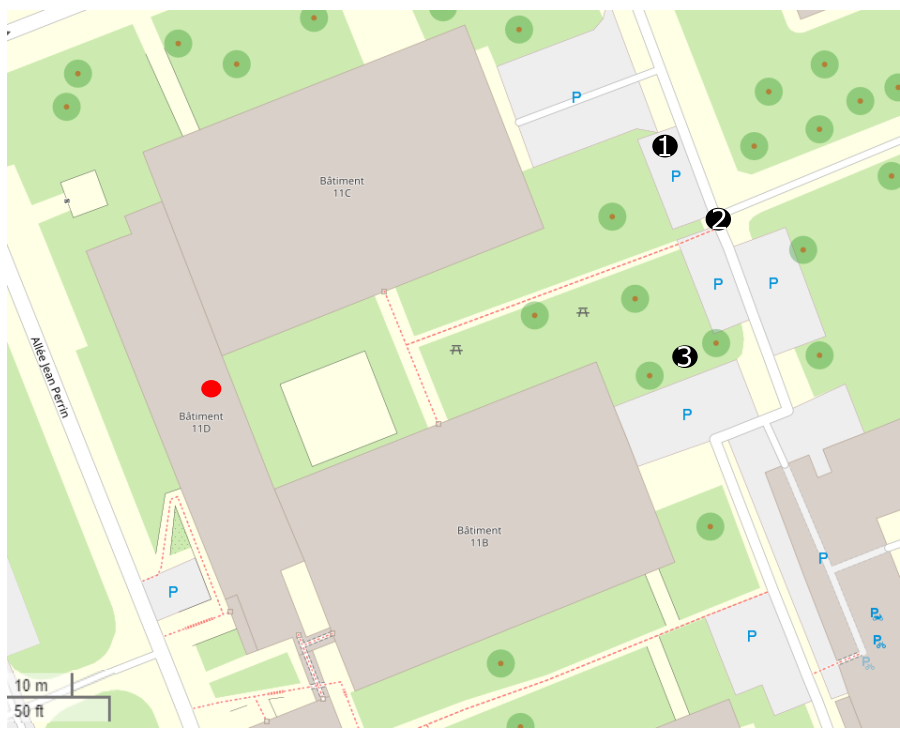

Fig. 1: The locations of the three measured points in the area of the Campus Beaulieu. Positions of Tx and the Rx are labeled by black, and red markers respectively. (Cby OpenStreetMap Contributers)

Whereas the aforementioned emulated preamble LoRa signal is generated using python from the $m^{\text {th }}$ cyclic time shift of the basic chirp such that $x[n]$ is expressed as:

$$
x_{m}[n]=\sqrt{\frac{1}{2^{S F}}} \exp \left(j 2 \pi \frac{\left(\left((m+n) \bmod 2^{S F}\right)-2^{S F-1}\right)^{2}}{2^{1-S F}}\right),
$$

with $m=0$ for the basic chirp symbol, while the Spreading Factor (SF) is chosen to be equal to 7 . Where $n$ depicts the sample index $n=0,1,2, \ldots, 2^{S F}-1$.

\section{DATA PROCESSING}

On the Rx side, the saved file is imported to be analysed for each center frequency $f_{k}$. Thus, the following main signal processing techniques are carried out with the same order.

\section{A. Frequency synchronization}

The frequency and time synchronization is applied at first, respectively. The objective of frequency synchronization is to establish the subcarrier orthogonality by correcting the phase as an initial step before applying any further processing [11]. Let define the Carrier Frequency Offset (CFO) by $\Delta f_{k}$ as being the difference between the up and down conversion frequencies. This CFO results in a phase offset $\Delta \phi_{k}=2 \pi \frac{\Delta f_{k}}{f_{s}}$ between two samples with the same index in consecutive upchirps. The residual part of this offset can be estimated by taking the average across the received entire symbol $y_{k}[n]$ as: 


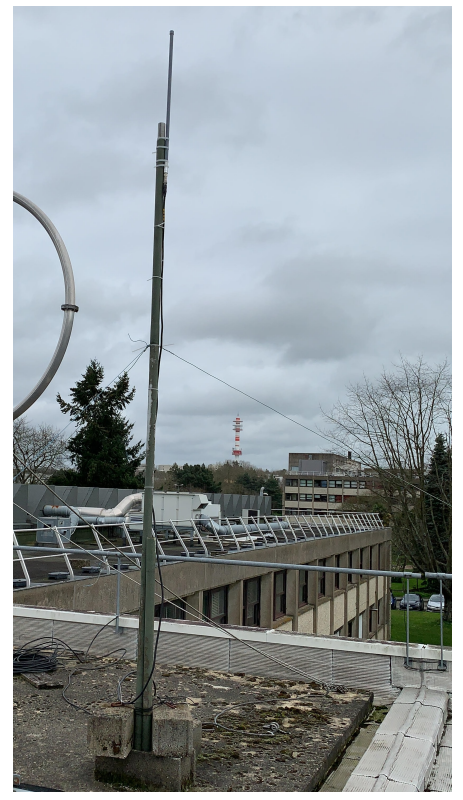

(a) Position of the Rx monopole antenna fixed on the roof.

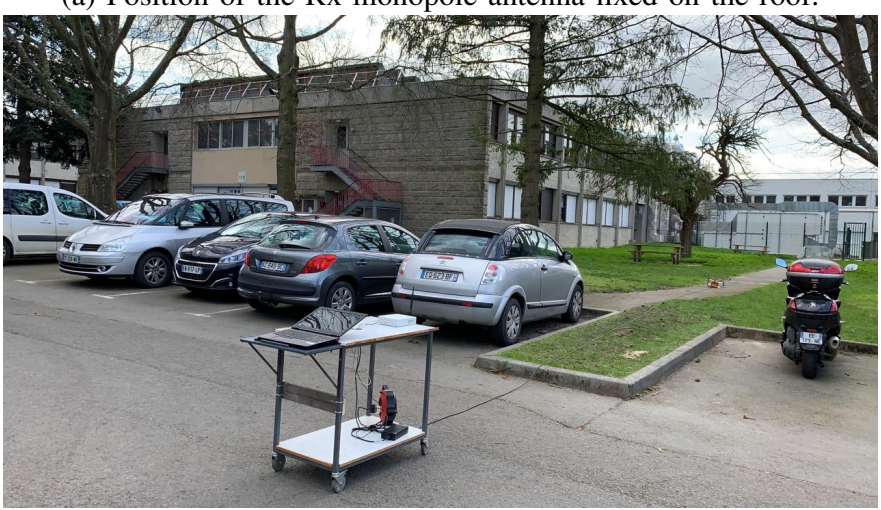

(b) A trolley shelf with the different parts of the transmission equipment at Tx location 2 .

Fig. 2: Views from the Tx and the Rx sites.

$$
\widehat{\Delta \phi_{k}}[l]=\arg \left(\sum_{n=0}^{2^{S F}-1} y_{k}[l+n] y_{k}^{*}\left[l+n+2^{S F}\right]\right) .
$$

This method of detecting the CFO is described as the frequency acquisition algorithm and is utilized over the whole handled signal portion. As shown in Figure 3, the angles of the differential correlation function indicate that there are some phase deviations. Hence, the phase error is compensated and the angles of the corrected differential correlation function become concentrated around zero.

\section{B. Channel estimation}

The up-chirps in the LoRa preamble, i.e. typically consist of eight symbols, are considered as a channel sounder. Therefore, the least squares estimate of the raw channel transfer function $\mathbf{H}_{\text {raw }}$ can be estimated for a simple division as [12]:

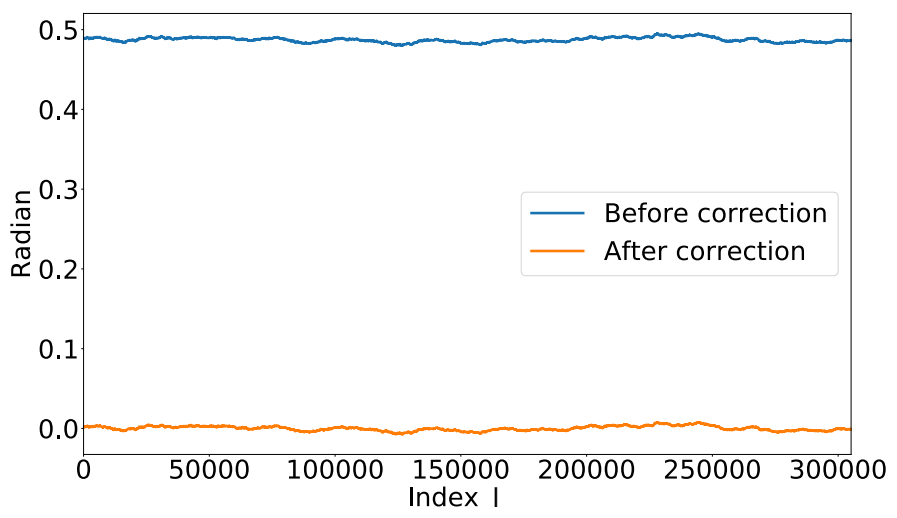

Fig. 3: The angles of differential correlation function $\widehat{\Delta \phi_{k}}[l]$ before and after correction of the whole portion of about 30 symbols.

$$
\mathbf{H}_{\text {raw }}=\frac{\mathbf{Y}}{\mathbf{X}},
$$

where $\mathbf{X}$ and $\mathbf{Y}$ are the training and the received symbol in the frequency domain respectively. Hence, the proposed denoising technique is applied on the raw channel transfer function $\mathbf{H}_{\text {raw }}$. This imposes doing an incipient step, i.e. removing the thermal noise from the subcarriers in the channel transfer function. Thus, the raw channel transfer function $\mathbf{H}_{\text {raw }}$ is low-passed in the frequency domain using FIR filter to maintain the property of linear phase as:

$$
\mathbf{H}_{\text {filtered }}=\mathbf{R}_{h} \cdot \mathbf{H}_{\text {raw }},
$$

where $\mathbf{R}_{h}$ is the autocorrelation matrix of the channel and the filtered channel $\mathbf{H}_{\text {filtered }}$ is also defined as $\mathbf{H}_{f_{k}}^{l}$. Where $f_{k}$ and $l$ are the center frequency value in $\mathrm{MHz}$ and the location number, respectively. As shown in Figure 4, one can observe that the obtained CSI is smooth without any noise at the three different locations. Moreover, it is obvious that the channels have a different magnitude of attenuation at the same subcarrier through the different locations.

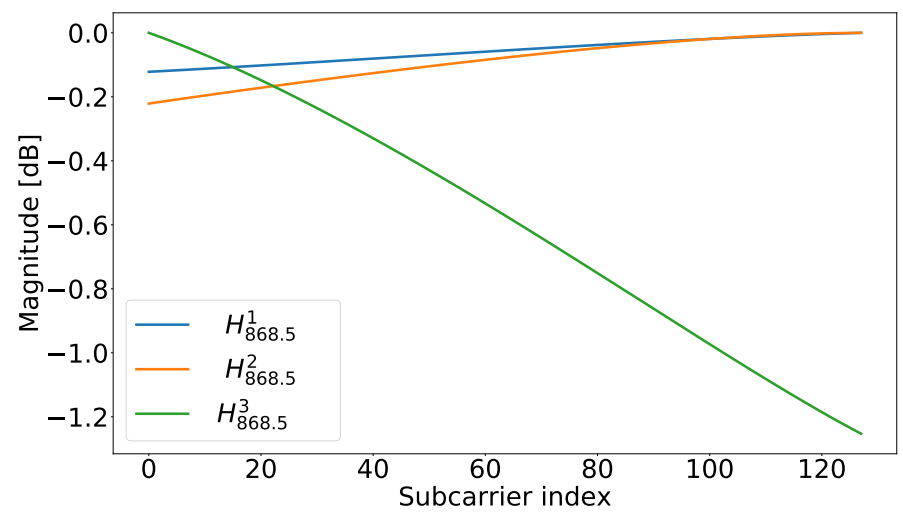

Fig. 4: The normalized CSI with $125 \mathrm{kHz}$ bandwidth. 


\section{CORRElation AnAlysis}

To check the plausibility of utilizing the CSI for localization, the CSI spatial and temporal evolution at each location is analysed using a proposed method based on the CSI slope. Furthermore, this technique is also compared to the traditional method which determines each position from its CSI average amplitude as detailed in the following subsections.

\section{A. CSI slope}

Based on the LoRa narrow-band characteristic, i.e. have a bandwidth $W=125 \mathrm{kHz}$ in Europe, a linear variation of the channel transfer function is assumed over frequency bandwidth centered on $f_{k}$. This condition is generally valid under the considered reality of such a very narrow bandwidth, thus, all the obtained channel transfer functions are almost flat as shown in Figure 4. Nevertheless, it is clear that each CSI has a unique slope. In this paper, the CSI slopes are estimated to analyse the CSI evolution from each location $i$ as:

$$
s_{f_{k}}^{i}=\frac{\left|\mathbf{H}_{f_{k}}^{i}[0]\right|-\left|\mathbf{H}_{f_{k}}^{i}\left[2^{S F}-1\right]\right|}{W},
$$

Then the Normalized Slope Distance (NSD) between any two positions for the whole $K$ uplink frequency bands is defined as:

$$
S_{i, j}=\frac{1}{2 K} \sum_{k=0}^{K-1}\left|\tilde{s}_{f_{k}}^{i}-\tilde{s}_{f_{k}}^{j}\right|
$$

with

$$
\tilde{s}_{f_{k}}^{i}=\frac{s_{f_{k}}^{i}}{S_{\max }}
$$

where $S_{\max }$ is equivalent to the absolute value of the maximum observed slope in the measured dataset. This is chosen to be the scaling factor for the slope normalization. Consequently,

$$
0 \leq S_{i, j} \leq 1
$$

The magnitudes of the aforementioned normalized slope $\tilde{s}_{f_{k}}^{i}$ are given in Figure 5a for each different location $i$ and center frequency $f_{k}$. Thus, the full scale is normalized and lies in $[-1,1]$, whereas every amplitude can vary on a several order of magnitude levels. Each frequency of the $K$ uplink frequency bands is labeled with a specific color. In order to analyse the time variation of the CSI slope at each position, over a time duration $\tau$ of about 10 minutes, an arrow is drawn for every center frequency from the first time instant to the second one. This measurement dataset demonstrates the slow alteration of the CSI slope with respect to time.

Moving to the NSD $S_{i, j}$ values in Figure 6a, one can observe the high level of NSD values in the three location combinations, which are $S_{1,2}, S_{1,3}$ and $S_{2,3}$. This indicates that CSI models from different positions are distinctly different. While the CSI for each location significantly underlines a low NSD value with itself after a $\tau$ time interval. This is obvious in $S_{1,1^{\tau}}, S_{2,2^{\tau}}$ and $S_{3,3^{\tau}}$ whose values are near zero as well as they are far away from the other NSD values, i.e. labeled by circles. So the environment of the propagation paths for each specific position is stable with only marginal modification during this duration.

\section{B. CSI average magnitude}

On the other hand, the channel evolution at each location is investigated by the mechanism, which depends on calculating the CSI mean magnitude. This method is considered as an alternative way rather than estimating the RSSI value. Thus, the CSI average amplitude is estimated for each location $i$ as:

$$
r_{f_{k}}^{i}=\frac{1}{2^{S F}} \sum_{n=0}^{2^{S F}-1}\left|\mathbf{H}_{f_{k}}^{i}[n]\right|,
$$

Hence, the Amplitude Distance (AD) between any two positions for the entire $K$ uplink frequency bands is computed as:

$$
R_{i, j}=\frac{1}{K} \sum_{k=0}^{K-1}\left|r_{f_{k}}^{i}-r_{f_{k}}^{j}\right|
$$

For the whole evaluated locations and frequency bands, the measured values of the CSI average amplitude $r_{f_{k}}^{i}$ are given in Figure 5b. It is foremost supposed that the CSI slope variability from one sub-band to another is more informative than in the CSI average magnitude whose amplitudes at various bands are evaluating roughly at near levels. Moreover, it seems that the CSI average amplitude has more alternation in time than the CSI slope, which is particularly clear at location 2 when considering the shift between the values of 2 and $2^{\tau}$.

As depicted in Figure $6 \mathrm{~b}$, the AD $R_{i, j}$ values obtained for all the location combinations preserve proper values. However, the $\mathrm{AD}$ values, i.e. labeled by triangles, for each location with itself after the time interval $\tau$ are near to the AD values of different location combinations, i.e. labeled by circles. This could be observed explicitly in the high AD value of $R_{2,2^{\tau}}$. This confirms the proposed hypothesis which asserts that the CSI is more robust to the signal fluctuations than the RSSI, because, the CSI average magnitude values don't have the same temporal stability as the CSI slopes.

\section{CONCLusions}

Rather than using RSSI fingerprinting, in this article, the feasibility of utilizing the CSI for localization is presented. This hypothesis intends to improve the accuracy of positioning by utilizing the rich channel information in each subcarrier as well as the frequency hopping in the LoRa systems. To allow a dynamic study of this approach, an outdoor measurement campaign is carried out in the area of the Campus Beaulieu in Rennes to estimate the CSI of transmitted LoRa signals 


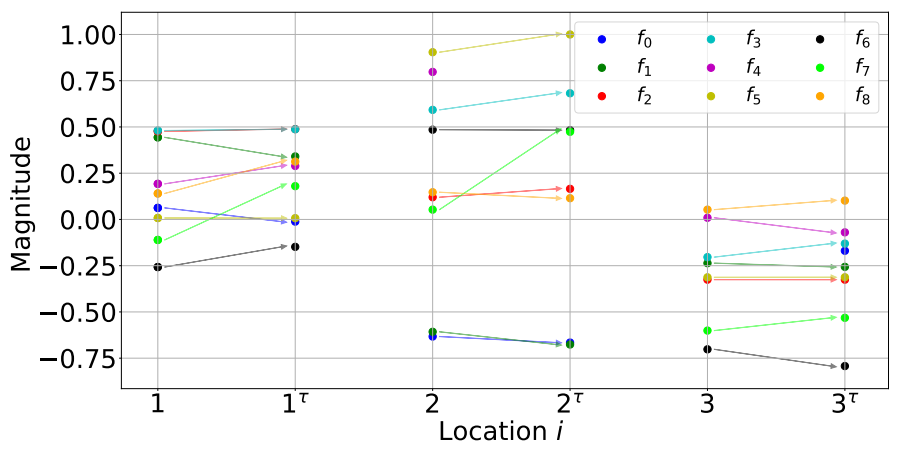

(a) The normalized slope $\tilde{s}_{f_{k}}^{i}$ values.

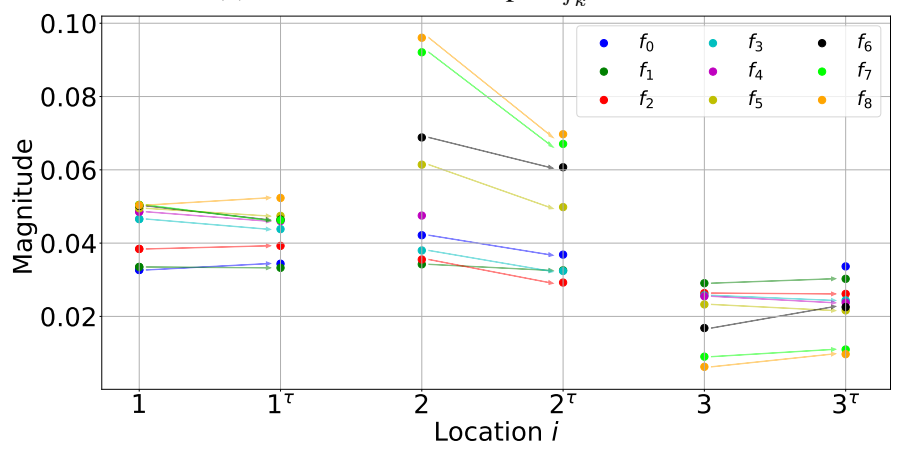

(b) The CSI average amplitude $r_{f_{k}}^{i}$ values.

Fig. 5: A comparison between the two families of the distinct observables.

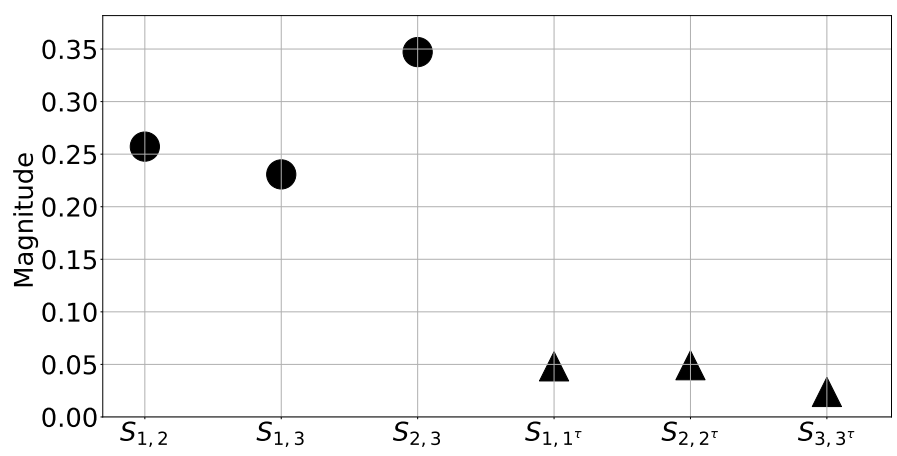

(a) The NSD $S_{i, j}$ values.

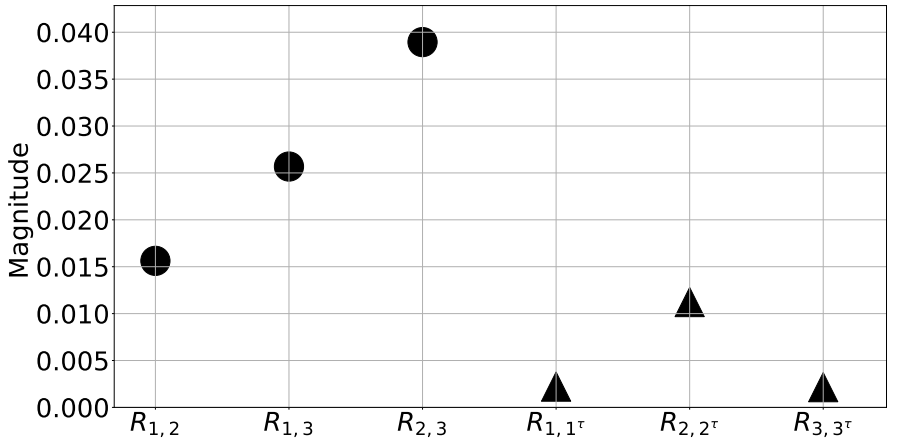

(b) The $\mathrm{AD} R_{i, j}$ values.

Fig. 6: The distance values. from different locations. Hence, the individual channels from each different location have to be appropriately different with one another to achieve significant localization gain. This difference in the CSI for every two locations is done based on two aspects, i.e. CSI slope and its average amplitude. Thus, the presented data indicate that CSI slopes are more stable and robust to the signal imperfections than the CSI average amplitudes. This result demonstrates the high performance of the CSI-based fingerprinting for positioning than RSSI, as well as its temporal stability.

In future work, CSI-based fingerprinting could be more efficient by using more than one gateway i.e., a realistic value in the near future, to obtain more than one CSI for the instant singular center frequency. Moreover, it could be improved with classical machine learning techniques for merging other radio observables such as RSSI, angle of arrival estimates or the propagation model.

\section{REFERENCES}

[1] M. Centenaro, L. Vangelista, A. Zanella and M. Zorzi, "Long-range communications in unlicensed bands: the rising stars in the IoT and smart city scenarios," in IEEE Wireless Communications, vol. 23, no. 5, pp. 60-67, October 2016.

[2] F. Adelantado, X. Vilajosana, P. Tuset-Peiro, B. Martinez, J. Melia-Segui and T. Watteyne, "Understanding the Limits of LoRaWAN," in IEEE Communications Magazine, vol. 55, no. 9, pp. 34-40, Sept. 2017.

[3] L. Mainetti, L. Patrono, A. Secco and I. Sergi, "An IoT-aware AAL system for elderly people," 2016 International Multidisciplinary Conference on Computer and Energy Science (SpliTech), Split, 2016, pp. 1-6.

[4] A. H. Sayed, A. Tarighat and N. Khajehnouri, "Network-based wireless location: challenges faced in developing techniques for accurate wireless location information," in IEEE Signal Processing Magazine, vol. 22, no. 4, pp. 24-40, July 2005.

[5] Z. Li, T. Braun, X. Zhao, Z. Zhao, F. Hu and H. Liang, "A Narrow-Band Indoor Positioning System by Fusing Time and Received Signal Strength via Ensemble Learning," in IEEE Access, vol. 6, pp. 9936-9950, 2018.

[6] Zafari, F., Gkelias, A., and Leung, K. K. (2019). A Survey of Indoor Localization Systems and Technologies. IEEE Communications Surveys and Tutorials, $1-1$.

[7] B. C. Fargas and M. N. Petersen, "GPS-free geolocation using LoRa in low-power WANs," 2017 Global Internet of Things Summit (GIoTS), Geneva, 2017, pp. 1-6.

[8] Choi, Wongeun and Chang, Yoon-Seop and Jung, Yeonuk and Song, Junkeun. (2018). Low-Power LoRa Signal-Based Outdoor Positioning Using Fingerprint Algorithm. ISPRS International Journal of GeoInformation.

[9] K. Wu, J. Xiao, Y. Yi, D. Chen, X. Luo and L. M. Ni, "CSI-Based Indoor Localization," in IEEE Transactions on Parallel and Distributed Systems, vol. 24, no. 7, pp. 1300-1309, July 2013.

[10] Q. Song, S. Guo, X. Liu and Y. Yang, "CSI Amplitude FingerprintingBased NB-IoT Indoor Localization," in IEEE Internet of Things Journal, vol. 5, no. 3, pp. 1494-1504, June 2018.

[11] R. Ghanaatian, O. Afisiadis, M. Cotting and A. Burg, "Lora Digital Receiver Analysis and Implementation,’ ICASSP 2019 - 2019 IEEE International Conference on Acoustics, Speech and Signal Processing (ICASSP), Brighton, United Kingdom, 2019, pp. 1498-1502.

[12] J. -. van de Beek, O. Edfors, M. Sandell, S. K. Wilson and P. O. Borjesson, "On channel estimation in OFDM systems," 1995 IEEE 45th Vehicular Technology Conference. Countdown to the Wireless TwentyFirst Century, Chicago, IL, USA, 1995, pp. 815-819 vol.2. 